\title{
A Dual UAV Docking System Based on Monocular Vision Ranging and Positioning
}

\author{
Junxing $\mathrm{Li}^{1}$, a, $\mathrm{Ce} \mathrm{Li}^{2}$, b , Liming $\mathrm{Wu}^{1, \mathrm{c}}$ and Yachun Zheng ${ }^{1, d}$ \\ ${ }^{1}$ School of Electromechanical Engineering, Guangdong University of Technology, Guangzhou \\ 510006, China; \\ 2State Key Laboratory of Coal Resources and Safe Mining, China University of Mining and \\ Technology, Beijing 100083, China.
}

ali-junxing@163.com, bcelicv@qq.com, cjkyjs@gdut.edu.cn, dzheng_yachun@163.com

\begin{abstract}
The dual UAV docking system is recently known as an important challenging task to improve the cruising ability of the multi-rotor UAV. In this paper, a mechanical structure of this system is illustrated. Monocular vision is adopted to identify the infrared beacons for the dual UAV charging positioning. The different effects of ultrasonic ranging and monocular vision ranging on this system are compared. Firstly, this system uses image processing to extract the characteristic values of the intercepts of the infrared beacon center and the actual position of the UAV, which are used to servocontrol the movement of the horizontal position of the mother UAV. The ultrasonic distance measurement and the monocular visual distance measurement are utilized to obtain the height differences between two aircrafts to implement charging and positioning. When the mother UAV entering in the docking range, the grapnel of the mother UAV is locked at the child UAV's bayonet to complete the docking mission. The experiment on actual platform testing shows the different effects of two ranging methods on the robustness of the system. The experiment results are compared to verify the correctness and effectiveness of my proposed method.
\end{abstract}

Keywords: Monocular vision, dual UAV docking system, ranging and positioning application.

\section{Introduction}

With the development of UAV navigation technology [1], multi-rotor UAVs have gradually been applied to aerial photography and the natural disaster scene for emergency rescue and disaster relief [2]. However, the UAV in the prior art can only meet the requirements of short-range and low-altitude. The problem of the life of the multi-rotor UAV has increasingly become a prominent issue that restricts the development of UAV technology.

To improve the cruising ability of the multi-rotor UAV, two UAVs are alternately used in the existing technology to meet the requirements of the UAV long range and high altitude. However, this method has problems of task interruption and inaccurate handover position. Aerial docking charging technology is a kind of UAV life extension technology that is under study [3]. This technology refers to the combination of a mother UAV and a child UAV through a docking mechanism to charge the child UAV during the child UAV's flight. The core of this technology lies in the positioning and docking of two UAVs.

At present, many types of research have been carried out on charging positioning and docking at home and abroad. Cassini et al. [4] designed a method for autonomous charging of a robot and a charging station through a light beam guide, which is suitable for an environment where the light intensity has a small influence in the room. Silverman et al. [5] used a laser distance meter and a visual identification beacon to charge and recharge the target. However, the laser distance meter is expensive and this method requires that the charging pin must face the charging station. The charging socket is small so the charging pin must be inserted directly. And it requires that the positioning accuracy must be high. Ren C. Luo et al. [6] used two microphones to track the sound source to guide the robot to autonomously charge. However, in the outdoor environment, the sound wave is easily divergent, and the speed of sound is susceptible to interference, and the docking accuracy is not high. Lin Dan et al. [7] proposed an autonomous return path planning strategy aiming at returning to a fixed charging station. This method requires that the robot has prior knowledge and does not apply to drone docking in an unfamiliar environment. 
This article adopts monocular vision to identify the infrared beacons for the dual UAV charging positioning, and compares the different effects of ultrasonic ranging and monocular vision ranging on this system. Firstly, this system uses image processing to extract the characteristic values of the intercepts of the infrared beacon center and the actual position of the UAV. These are used to servocontrol the movement of the horizontal position of the mother UAV. The ultrasonic distance measurement and the monocular visual distance measurement are used to obtain the height differences between two aircrafts to implement charging and positioning. When the mother UAV entering the docking range, the grapnel of the mother UAV is locked at the child UAV's bayonet to complete the docking mission.

\section{System Module and Mechanical Structure}

In this paper, the child UAV is similar to the main part of the mother UAV. The flight control adopts the attitude sensor to estimate the current attitude of the fuselage, and sends the signal from the flight controller to the electronic governor to control the rotation speed of the motor as shown in Fig.1. The camera module is installed at the bottom of the mother UAV, and the infrared beacon module of the child UAV in the image is identified by the image processing module to perform position servo control. After entering the docking range, the grapnel of the mother UAV is locked at the child UAV's bayonet as shown in Fig.2. A pressure strain gauge for sensing whether the grapnel lock the bayonet is provided at the bayonet [8]. The control system used in this article is referred to [9], which includes attitude control and position control.

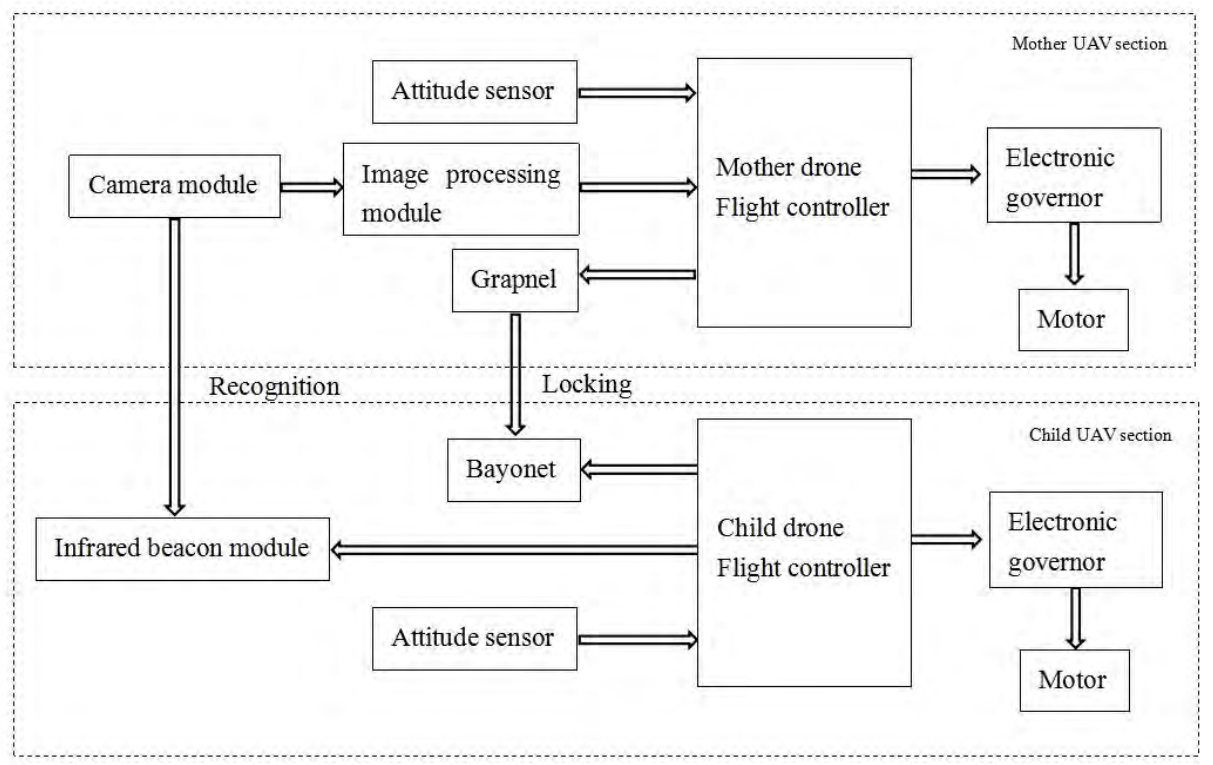

Fig.1 Module diagram of UAV in dual UAV docking system

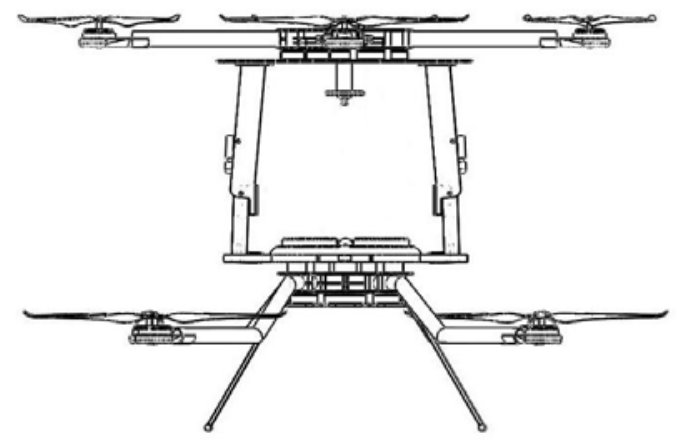

Fig.2 Schematic diagram of UAVs' structure after docking 


\section{Visual Recognition Algorithm}

In the practical application of color block recognition, the accuracy of recognition of the color block is easily affected by ambient lighting, shooting angles, and other similar color blocks in the background. In order to increase the recognition success rate, this system uses a camera equipped with a high-pass optical filter to identify infrared LED beacon to reduce the interference of other objects in the background. At the same time, the infrared LED beacon is an active optical beacon whose brightness is less affected by the ambient light, thereby reducing the difficulty of the beacon recognition of this system and improving the recognition accuracy.

\subsection{Grayscale Image Segmentation Algorithm.}

After the ambient light is filtered out by the optical filter, the background object is basically black. The traditional segmentation algorithm for grayscale image can be mainly divided into three types: edge detection type, threshold type, and region detection type. The recognition target and background of this system have the large difference in grayscale. Therefore, a threshold detection method with high computational efficiency and high speed is used [10].

In this paper, a threshold range is set for the gray value in the grayscale image, and any image pixel that meets the threshold range is the target pixel within the color block. The original grayscale image brightness is $f(x, y)$. By dividing the threshold, the image is divided into two parts. Then the brightness of the segmented binary image $g(x, y)$ is shown in Eq. (4). The $t_{-}$upper and

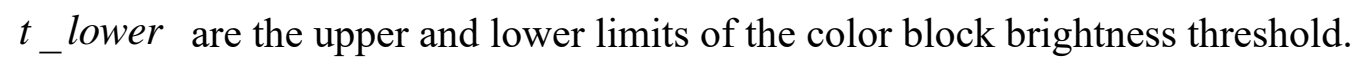

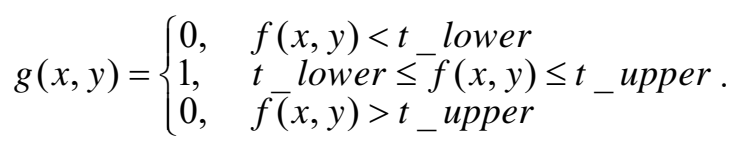

\subsection{Infrared Beacon Minimum Bounding Rectangle.}

In the image processed above, the infrared beacon consists of a set of white pixels. In this paper, we need to outline a minimum bounding rectangle for the edge of the pixel block to calculate the size of the beacon area and the position of the beacon center. We set the coordinates of this set of white pixels to $\left(x_{i}, y_{i}\right)(i=1,2, \ldots, n)$. The pixel coordinate of the upper left corner of the rectangle is $\left(x_{l u}, y_{l u}\right)$. The pixel coordinates in the lower right corner of the rectangle is $\left(x_{r l}, y_{r l}\right)$. The formula for the two points is

$$
x_{l u}=\min \left\{x_{i}\right\}, y_{l u}=\min \left\{y_{i}\right\}, x_{r l}=\max \left\{x_{i}\right\}, y_{r l}=\max \left\{y_{i}\right\}, i \in\{1, \ldots, n\} .
$$

The upper left corner and the lower right corner of the rectangle can be drawn to obtain the four vertices of the rectangle. The four vertices of the rectangular box are connected to obtain the minimum bounding rectangle. Select the center point of the target's minimum bounding rectangle as the beacon center point as shown in Fig.3. When this point is identified, it will be used as the target point of the UAV.

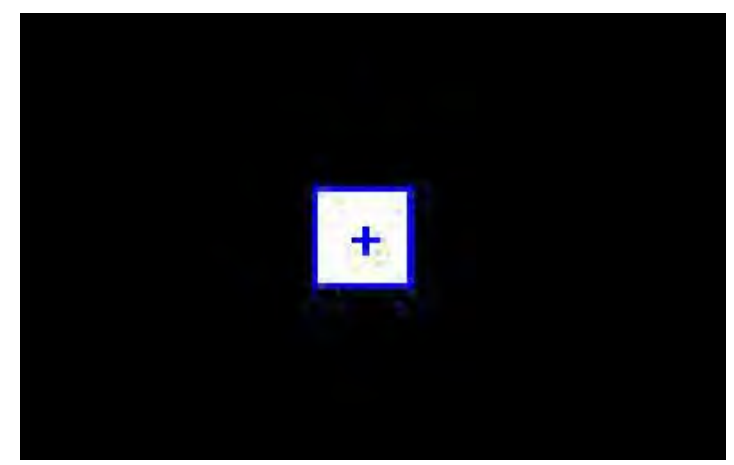

Fig.3 The bounding rectangles for two examples of infrared beacon 


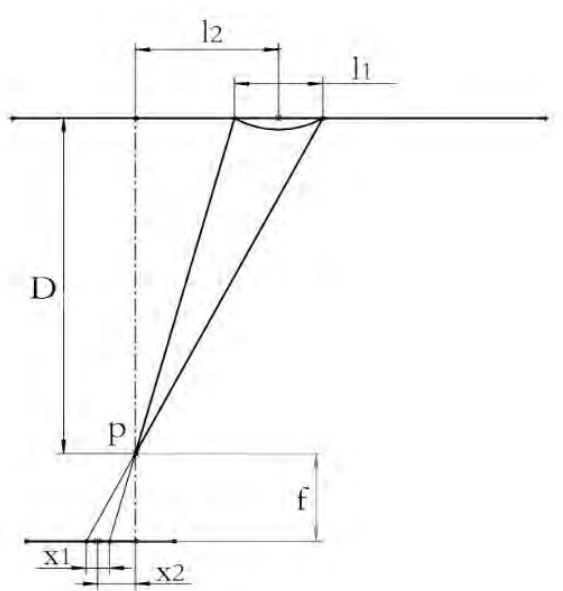

Fig.4 Monocular vision ranging schematic

\section{UAV Height Measurement}

Height data is an important parameter for UAV flight control. In this paper, the dual UAV docking system needs to obtain the exact relative height of the two UAVs for height control. At the same time, in order to replace the pixel number deviation obtained by the visual recognition system with the actual distance deviation, we also need to obtain an accurate relative height. The existing UAV ranging methods include laser radar ranging, ultrasonic ranging, and visual ranging. Laser radar ranging requires the support of high-precision special hardware, and the cost of laser radar is high. Ultrasonic ranging [11] is superior to laser radar ranging in control method, cost performance and safety. However, ultrasonic ranging faces a problem in this system, and the ultrasonic module can only obtain the distance between the drone and the obstacle directly below. When the mother UAV approached the child UAV, ultrasonic waves were applied to the fuselage of the child UAV, and the measured distance suddenly changed to the relative distance of the two UAVs. In contrast, the monocular vision ranging method is low in cost and high in flexibility. Using visual positioning can achieve accurate positioning between two UAVs. This article uses monocular vision ranging, that is, only one camera is used to obtain the image and calculate the distance deviation from the target.

Monocular vision ranging means that only one camera is used to obtain the image and calculate the distance deviation from the target [12]. In this article, a model based on the spatial parallel plane constraint is used. As shown in Fig.4, we take the measurement in the x-axis direction of the image coordinate system as an example. Let the focus of the camera be the point $\mathrm{P}$, the focal length of the camera $f$, and the actual side length of the square infrared beacon $l_{1}$. Assume that the side length of the square infrared beacon on the image plane is $x_{1}$. The distance between the center of the square infrared beacon and the center of the image plane on the image plane is $x_{2}$. The actual distance between the projection point of the fuselage in the plane of the infrared beacon and the center point of the infrared beacon is $l_{2}$. Usually we ignore the thickness of the body and the thickness of the lens, and assume that the distance from the center of the camera to the plane of the infrared beacon is $D$. According to the similar principle of triangles, the problem is formulated as:

$$
\left\{\begin{array}{l}
\frac{l_{2}}{D}=\frac{x_{2}}{f} \\
\frac{l_{2}-\frac{l_{1}}{2}}{D}=\frac{x_{2}-\frac{x_{1}}{2}}{f}
\end{array} .\right.
$$

And we have: 


$$
D=\frac{l_{1} \cdot f}{x_{1}}
$$

\section{Visual Servo Positioning Based on Image Processing}

After the mother UAV approaches the child UAV, the child UAV enters the field of vision of the mother UAV's camera. The dual UAV docking system needs precise positioning [13]. According to the attitude angle of the UAV, the actual position deviation of the UAV and the infrared beacon is obtained by the algorithm. First, consider the influence of only the roll angle $\phi$, the visual positioning geometric relationship is shown in Fig.5. The drone flew over the infrared beacon with a roll angle $\phi$.

Suppose the camera parameters are known, the lens focal length is $f$, the roll angle is $\phi$, the actual position of the fuselage is point $\mathrm{R}$. The actual position of the fuselage on the image plane is point $\mathrm{B}$, the actual position of the infrared beacon is point $\mathrm{E}$, and the projection point of the infrared beacon on the image plane is point $\mathrm{C}$. Assume that the actual distance from point $\mathrm{A}$ to point $\mathrm{C}$ is $L_{A C}$. $L_{A C}$ can be calculated in image plane coordinate system [14]. The distance from point $\mathrm{R}$ to point $\mathrm{E}$ is the deviation $L_{X}$ between the actual position of the fuselage and the actual position of the infrared

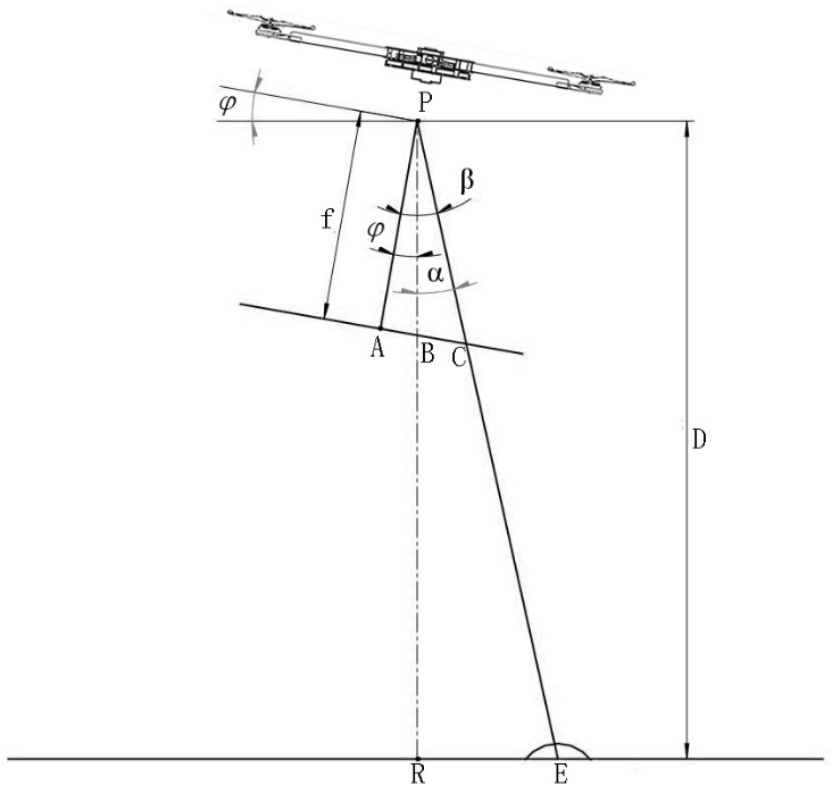

Fig.5 Monocular vision positioning principle schematic.

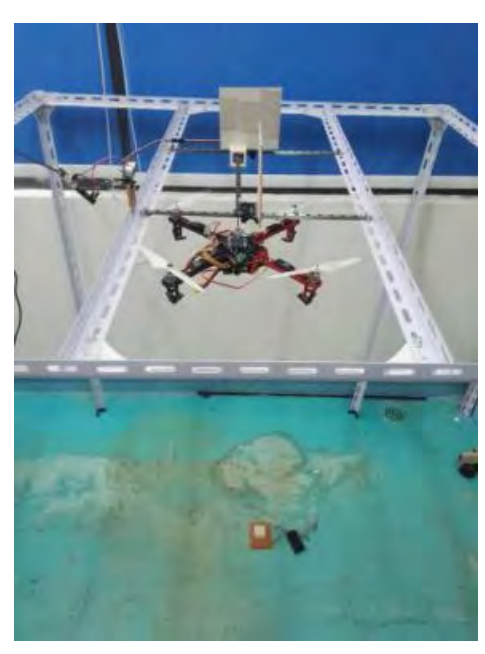

Fig.6 Experiment scene of actual flight.

beacon in the $\mathrm{x}$-axis direction of the ground coordinate system. Ignoring fuselage thickness and lens thickness. From Section 4, the height D of the UAV to the plane where the infrared beacon is located can be obtained. The trigonometric function is solved by:

$$
\begin{gathered}
\beta=\arctan \left(\frac{L_{A C}}{f}\right), \\
\alpha=\beta-\phi, \\
L_{X}=D \cdot \tan \alpha .
\end{gathered}
$$

Similarly, considering the pitch angle $\theta$, the deviation $L_{Y}$ between the actual position of the body and the actual position of the infrared beacon in the y-axis direction of the ground coordinate 
system can be obtained. Assume that the maximum horizontal error distance of the mother UAV relative to the child UAV is $S$. The $S$ depends on the design of the grapnel of the mother UAV. The visual positioning servo-control rule is that by controlling the positional deviation of the mother UAV relative to the child UAV, the distance deviation $L_{X}$ and $L_{Y}$ tend to 0 . When the distance deviation satisfies $-S<L_{X}<S$ and $-S<L_{Y}<S$, it is considered that the mother UAV is aligned with the child UAV and the positioning of the dual UAV docking system is completed in the horizontal direction.

\section{Experimental Analysis}

\subsection{Experimental Design.}

In this article, the system acquires image information through the OpenMV3 module, processes it through relevant algorithms, and transmits the parameters to the flight control module through the serial port. The camera uses a $3.6 \mathrm{~mm}$ focal length lens and the front of the lens is equipped with $800 \mathrm{~nm}-2500 \mathrm{~nm}$ high-pass filter. The square infrared beacon uses nine infrared LEDs with a diameter of $10 \mathrm{~mm}$ and a wavelength of $850 \mathrm{~nm}$ to form a $3 \times 3$ LED matrix.

The beacon length is $35 \mathrm{~mm}$. A textured paper is placed above the infrared beacon to prevent the infrared beacon from becoming too bright, causing glare to the camera. The flight test platform is a quadrilateral bracket with a length of $1.2 \mathrm{~m}$, a width of $1.2 \mathrm{~m}$ and a height of $1.2 \mathrm{~m}$. There are three linear guides on the upper part of the bracket, allowing the slider to slide in the horizontal direction. The drone and the slider are connected through a universal joint, and the universal joint structure allows the UAV to tilt the fuselage to avoid interference with normal flight. The movement of the UAV will be transmitted to the slider through the universal joint, and the horizontal displacement data of the UAV will be measured through two ultrasonic ranging modules.

Table 1. Drone model parameters

\begin{tabular}{|c|c|c|}
\hline Name & Parameter Description & Value \\
\hline$m$ & Total mass of aircraft $/ \mathrm{kg}$ & 1.10 \\
\hline$l$ & Distance between the fuselage center and the motor $/ \mathrm{m}$ & 0.20 \\
\hline$I_{X}$ & $\mathrm{X}$-axis moment of inertia $\left./ \mathrm{kg} \cdot \mathrm{m}^{2}\right)$ & 0.114 \\
\hline$I_{Y}$ & $\mathrm{Y}$-axis moment of inertia/ $\left./ \mathrm{kg} \cdot \mathrm{m}^{2}\right)$ & 0.114 \\
\hline$I_{Z}$ & $\mathrm{Z}$-axis moment of inertia $\left./ \mathrm{kg} \cdot \mathrm{m}^{2}\right)$ & 0.158 \\
\hline
\end{tabular}

\subsection{System Actual Experiment}

The actual experiment of this system is performed on the test platform described above, as shown in Fig.6. The image resolution and frame rate are 120x160 and $20 \mathrm{fps}$, respectively. In this experiment, the UAV fuselage suspension height is $80 \mathrm{~cm}$, the initial coordinate is set to $(0,0)$, the beacon coordinate is $(150,150)$, and the unit is $\mathrm{mm}$. Because the test platform of this experiment adopts universal joint structure to connect with the UAV, and the linear guides have resistance. Therefore, the test platform can only reflect the approximate flight trajectory of the UAV, and cannot accurately reflect the small fluctuations in flight. At the same time, the speed of movement of the UAV is slightly affected by the resistance of the linear guides. However, this experiment is used to compare the system response under the two ranging methods. The above effects will also affect two ranging methods instead of one. Through comparative analysis of the approximate flight path, there are no strict requirements for small fluctuations in flight, so the analysis of conclusions is not affected.

First, the system test under the monocular vision ranging was performed. The test time was 20 seconds. The $\mathrm{x}$-axis trajectory and the $\mathrm{y}$-axis trajectory are obtained. Then perform system test under ultrasonic ranging with a test time of 20 seconds. With flight control settings, the measured height is set to twice the actual altitude within $0-1$ seconds after system was activated. It is used to simulate 
the effect that the height of the UAV from the ground is twice the height from the beacon. A comparison of the system responses under the two ranging methods is shown in Fig.7.

In Fig.7, we can see that under the monocular vision ranging, the system has a small overshoot and it is smoother, more stable, and reaches a steady state faster. But whether it is the x-axis trajectory or the y-axis trajectory, the tracking of the beacon eventually still has a static error. This may be caused by the error of the test platform or the recognition error caused by the resolution of the camera. In the case of ultrasonic ranging, the system is added with interference and a greater degree of overshoot occurs. The convergence rate is faster, but there is still a small degree of shock. The time to reach steady state is still long, and the tracking of beacon eventually has static error.

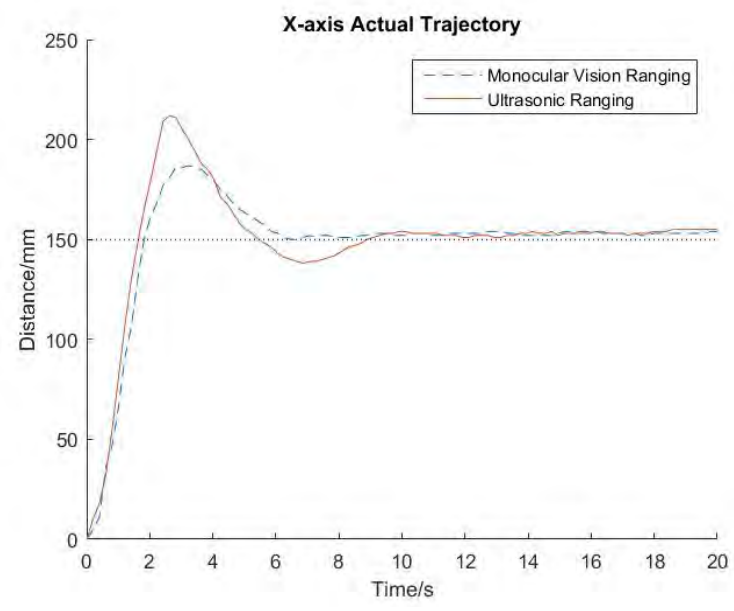

(a)

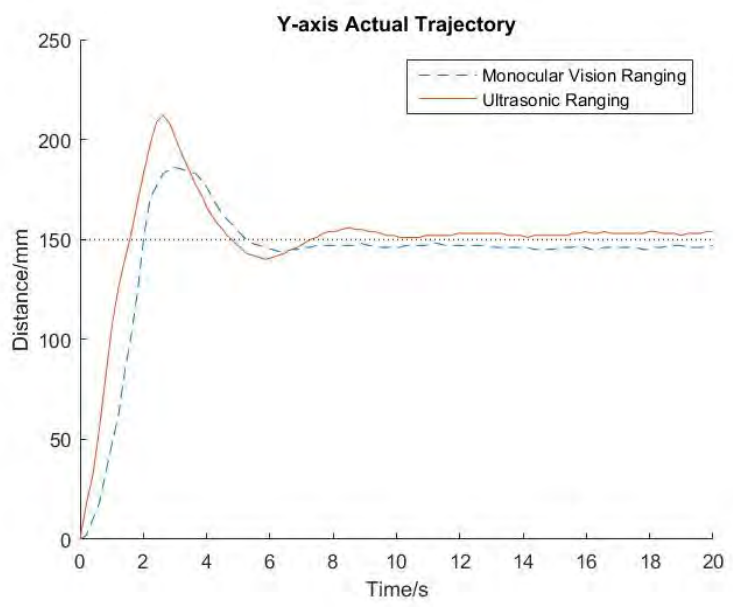

(b)

Fig.7 Illustration of (a) x-axis actual trajectory and (b) y-axis actual trajectory.

\section{Conclusion}

A dual UAV docking system based on monocular vision ranging and positioning is proposed in this paper, then the different effects of ultrasonic ranging and monocular vision ranging on this system are compared for verifying the UAV's ability to track infrared beacon and compare the impact of two ranging methods on system robustness. Image processing is used to extract the characteristic values of the intercept points of the infrared beacon center and the actual position of the UAV to servocontrol the movement of the horizontal position of the UAV. Through the actual platform testing, the different influences of the two ranging methods on the robustness of the system are compared. It shows that the monocular vision ranging can avoid the range mutation caused by ultrasonic ranging, and the system is more robust under the visual ranging method. It is proved that the monocular vision ranging can provide a reasonable ranging and associated control feedback, and that future hardware tests of the grapnel of the mother UAV are intended to show that the monocular vision ranging and positioning approach in dual UAV docking system is feasible. The experimental results demonstrate that my dual UAV docking system can improve the correctness and effectiveness.

\section{Acknowledgements}

The work was supported by the State Key Laboratory of Coal Resources and Safe Mining under Contract SKLCRSM16KFD04. The work was also supported in part by the Fundamental Research Funds for the Central Universities (2016QJ04), and the Yue Qi Young Scholar Project.

\section{References}

[1]. Kendoul, F. Survey of advances in guidance, navigation, and control of unmanned rotorcraft systems. Journal of Field Robotics, Vol. 29 (2012) No. 2, p. 315-378. 
[2]. Ren, J., Gao, X. Situation Assessment Model for UAV Disaster Relief in the City. In 2011 International Workshop on Multi-Platform/Multi-Sensor Remote Sensing and Mapping, 2011, p. $1-6$.

[3]. Shenzhen Flyeah Intelligent Technology co.LTD. A Dual UAV System with an Air Charging Lift Capability, Chinese Patent NO.CN201510704012.7[P]. 2016-01-27.

[4]. Cassinis, R., Tampalini, F., Bartolini, P., et al. Docking and Charging System for Autonomous Mobile Robots, 2005.

[5]. Silverman, M. C., Nies, D., Jung, B., et al. Staying alive: a docking station for autonomous robot recharging. In Proceedings 2002 IEEE International Conference on Robotics and Automation (Cat. No.02CH37292) Vol. 1, 2002, p. 1050-1055.

[6]. Luo, R. C., Huang, C.-H., Huang, C.-Y. Search and track power charge docking station based on sound source for autonomous mobile robot applications. In 2010 IEEE/RSJ International Conference on Intelligent Robots and Systems, 2010, p. 1347-1352.

[7]. Lin, D. A Return Docking Algorithm for Indoor Cleaning Robot. Journal of Chongqing University of Science and Technology, 2010, Vol. 1, p. 110-113.

[8]. Guangdong University of Technology. A Dual UAV Docking System for Air Docking and Batteries Replacement, Chinese Patent NO.CN201810044053.1[P]. 2018-05-18.

[9]. Sheng, Z. Vison-based pose control and autonomous return for an unmanned aerial vehicle. Doctoral dissertation, Shanghai Jiao Tong University, 2015.

[10]. Lin, K., Wu, J., Xu, L. A Survey on Color Image Segmentation Techniques. Journal of Image and Graphics, Vol. 10, 2005, No. 1, p. 1-10.

[11]. Ishihara, M., Shiina, M., Suzuki, S. Evaluation of Method of Measuring Distance Between Object and Walls Using Ultrasonic Sensors. Journal of Asian Electric Vehicles, Vol. 7, 2009, No. 1, p. 1207-1211.

[12]. Xiao-ning, F. Study on Distance Measurement Based on Monocular Vision Technique. Journal of Shandong University of Science and Technology, 2007.

[13]. Hutchinson, S., Hager, G. D., Corke, P. I. A tutorial on visual servo control. International Conference on Robotics and Automation, Vol. 12, 1996, No. 5, p. 651-670.

[14]. Li, Q. Research on monocular vision-based realtime distance measurement algorithm. Doctoral dissertation, Harbin Institute of Technology, 2014. 